\title{
Research on Resource Scheduling Method Based on Improved Hungary Algorithm
}

\author{
Tingpeng Li \\ Science and Technology on Integrated Logistics \\ Support Laboratory, \\ National University of Defense Technology, \\ Changsha, China \\ E-mail: tovey1987@126.com
}

\section{Yue Li}

Science and Technology on Integrated Logistics Support Laboratory, National University of Defense Technology, Changsha, China

E-mail: liyue@ nudt.edu.cn

\author{
Yanling Qian \\ Science and Technology on Integrated Logistics \\ Support Laboratory, \\ National University of Defense Technology, \\ Changsha, China \\ E-mail: ylqian@ nudt.edu.cn
}

\section{Bin $\mathrm{Li}$}

Science and Technology on Integrated Logistics Support Laboratory, National University of Defense Technology, Changsha, China

E-mail: libing@ nudt.edu.cn

\begin{abstract}
The optimal allocation of resources is of great significance to improve the efficiency and reduce the cost of equipment manufacturing. For the shortage of traditional Hungary algorithm that cannot solve the resource scheduling problem containing the parallel jobs, an improved Hungary algorithm is proposed in this paper. The improved algorithm converts the problem to a typical assignment problem by replacing parallel link with virtual job. And then, optimizing it with classical Hungary algorithm and determining the realizability of the virtual job based on the result. Finally, the optimal scheme will be got through iterative searching. In addition, an example of resources allocation optimizing of an equipment manufacturing system is introduced to verify the effectiveness of the proposed algorithm.
\end{abstract} $\begin{array}{cccc}\text { Keywords- } & \text { Hungary } & \text { Algorithm; } & \begin{array}{r}\text { Equipment } \\ \text { Manufacturing; }\end{array} \\ \text { Resource } & \text { Scheduling; } & \text { Virtual Job; }\end{array}$ Assignment Problem

\section{INTRODUCTION}

Task assignment is a classic resources optimization schedule problem $-m$ workers are assigned to deal with $n$ jobs and the efficiency of workers is different, determining the task assignment scheme to get the best total efficiency. Because of simple steps and ability to get the optimal solution without validation, Hungary algorithm is widely used in the assignment problem [1].

In this paper [2], based on the analysis of classical Hungary algorithm, an improved Hungary algorithm, "add zero row method", is proposed to solve the incomplete assignment problem. In reference [3], MATLAB program of Hungary algorithm is introduced and used to solve the typical assignment problem, such as marriage assignment. Literature [4] proposed a new method, "difference method", to solve the non-standard assignment problem. Compared with the traditional algorithm, the method is more simple and intuitive. In this paper [5], Hungary method is applied to solve the abnormal flight emergency scheduling problem and good results have been achieved. Literature [6] proposed an improved Hungary algorithm and used it to study the multiple maintenance scheduling problems with harsh environments. Literature [7] studied the Unmanned Aerial Vehicle (UAV) target assignment problem with Evolution Hungary algorithm. In this paper [8], Hungary algorithm and topology constraints are combined to research the cell tracking problem under the condition of high density. In this paper [9], in order to improve the efficiency of the distribution of the cloud computing tasks, based on the standard Hungary algorithm, a fast order reduction optimization algorithm is proposed. By eliminating the matrix elements which are decided, the order of the matrix will be reduced quickly and the computational efficiency is improved. Literature [10] studied the dynamic power allocation of weapon - target with Hungarian method by transforming it into an assignment problem.

Although Hungary algorithm has been used in many domains and achieved good results, the traditional Hungary algorithm can only solve the problems which the total cost is the sum of each task. However, in practical engineering, many problems do not satisfy the condition. Therefore, the algorithm should be improved so as to solve those problems.

\section{THE CLASSICAL HUNGARY ALGORITHM AND ITS SHORTAGE}

\section{A. The classical Hungary algorithm.}

Hungary algorithm is an optimization method proposed by W. W. Kuhn to solve the assignment problem with the independent zero elements theory about matrix proposed by Hungarian mathematician D. Koning [6]. The theoretical basis of the method is that any row or column of the efficiency matrix (also called cost matrix) add or minus a constant doesn't change the optimal allocation scheme [11]. The basic idea is to modify the efficiency matrix through adding or subtracting the same constant in each row or column until the matrix has at least one zero 
elements in different rows and different columns, and the zero elements can be used to carry out an optimal allocation scheme with minimum cost.

The detail steps of the classical Hungarian algorithm are shown as follows:

Step 1, establish the efficiency matrix M0 $(m * n)$ of resource allocation problem, where $m$ represents the number of workers and $n$ represents the number of jobs.

Step 2, in order to ensure every row has one zero element at least, every element of M0 minus the smallest element of its row. The modified matrix is marked as M1.

Step 3, in order to ensure every column has one zero element at least, every element of M1 minus the smallest element of its column. The modified matrix is marked as M2.

Step 4, cover all the zero elements of M2 with the least beeline. If the least amount of the lines is equal to $\mathrm{m}$, go to step 6, otherwise go to step 5. The modified matrix is marked as M3.

Step 5, all the elements of matrix M3 which are not covered by straight lines minus the smallest element, and every element which is covered by two straight lines adds the smallest element. The modified matrix is marked as M4 and M2 = M4, go to step 4.

Step 6, assign all the tasks start from the row or column which has least zero elements and the optimal assignment scheme is got.

In above steps, there is an assumption that $m$ equals to $\mathrm{n}$, that is to say, efficiency matrix M0 is a square matrix. But in the engineering applications, generally, the task number and the person number is not equal. In order to solve these problems (called incomplete assignment problem), the general approach is to add virtual person or virtual task to transform to a complete assignment problem. The detail method is introduced in references [1].

\section{B. The shortage of classical Hungary algorithm.}

Although it has been used in engineering application widely and achieved good results, the classical traditional Hungary algorithm can only solve the problems which the total cost is the sum of each task. If the cost is the work time of each task, the traditional Hungary algorithm can only solve the task assignment problem of series system which the total time is the sum of each task. However, in engineering practice, especially in the equipment manufactory system, series and parallel link exists at the same time is a very common situation. For example:

Question 1: there are four jobs needs to be completed and the order of jobs is shown as Fig.1. The work time of four workers to finish each job is known and every worker can only complete one task, the question is how to arrange the four workers to get the minimum time.

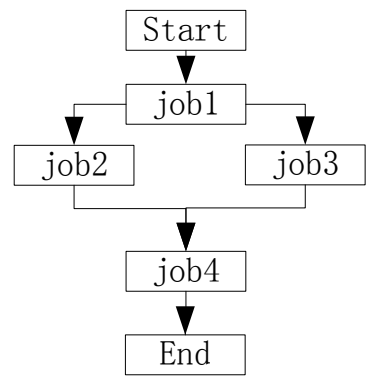

Figure 1. An Example of Manufactory System
Because only the maximum time of job 2 and job 3 affects the total time, the traditional Hungary algorithm cannot be used to solve this problem directly. In order to solve the problems like Question 1, the traditional Hungary algorithm must be improved.

\section{THE IMPROVED HUNGARIAN ALGORITHM}

The main reason of that traditional Hungary algorithm cannot solve the problems like Question 1 is that the total time of parallel link is not the sum of each job but only decided by the maximum one. The key way to solve the problem is transforming it to a classical assignment. Firstly, the series-parallel system is divided into two parts - series part and parallel part. Secondly, the parallel part is instead by a virtual job. Thirdly, the traditional Hungary algorithm is used to solve the new system. Based on the results, judging whether the virtual job can be implied by a parallel link which is consist of the idly workers and the worker who is assigned to the virtual job. If not, increase the time of the virtual job and try again, otherwise, the best assignment scheme is got.

\section{A. The detail steps of the improved Hungary algorithm}

In order to describe the improved Hungary in detail, this paper designs the detail steps of the algorithm.

Step 1, divide the system into series part and parallel part. If there are multiple parallel links, every parallel link is divided into an independent part.

Step 2, use a virtual job instead of parallel part, and initialize the work time of each worker as the Minimum Virtual Work Time (MVWT) which is the minimum time of the parallel part when the worker must finish one.

Step 3, establish the efficiency matrix $M$ with the jobs of series part and the virtual job. Based on $M$, the best assignment plan $\mathrm{P}$ is got with traditional Hungary algorithm.

Step 4, judge the virtual job of plan $\mathrm{P}$ whether can be instead of a parallel link which consist of the idly persons and the person who is assigned to the virtual job, if not go to step 5, otherwise go to step 6.

Step 5, a new virtual job is got by increase the work time of the old one and go to step 3.

Step 6, the final optimal allocation scheme $\mathrm{P}^{*}$ is got though instead of the virtual job with realizable parallel part.

\section{AN EXAMPLE}

In order to verify the effectiveness of the algorithm proposed in this paper, an example of task scheduling for an equipment manufacturing system is introduced.

\section{A. Problem description}

An equipment manufacturing system consists of 7 jobs and the flow diagraph is shown as Fig .2. In the system, job J3, job J4 and job J5 is a parallel link. The total work time is Tall:

$$
T_{\text {all }}=T_{1}+T_{2}+\max \left(T_{3}, T_{4}, T_{5}\right)+T_{6}+T_{7}
$$

Where, $T_{\mathrm{i}}(i=1,2, \cdots, 7)$ represent the work time of job Ji respectively 


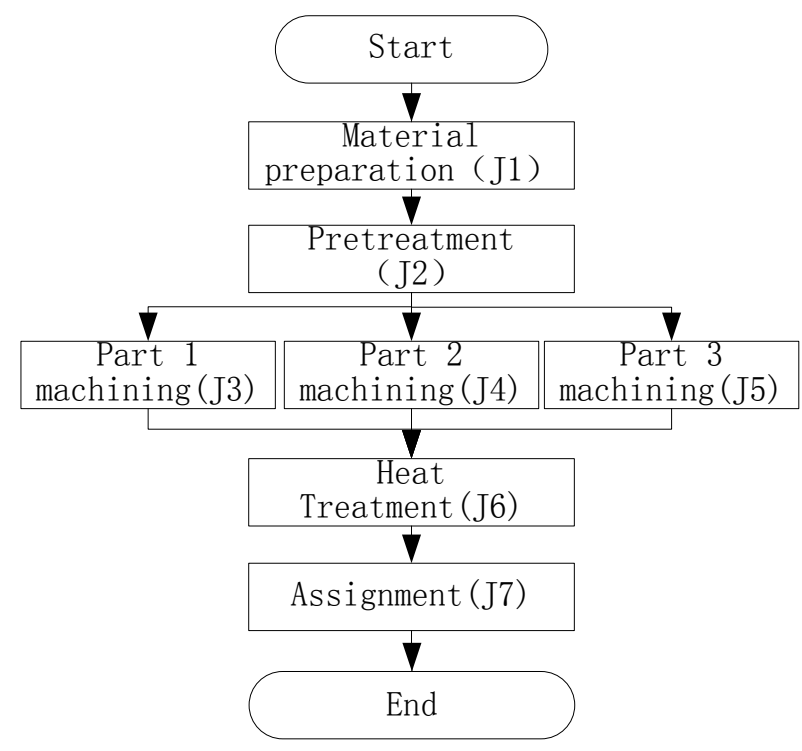

Figure 2. the flow diagraph of an equipment manufacturing system

There are 7 workers and the time of every worker to finish the jobs is shown as table I.

TABLE I. THE FINISH TIME OF JobS

\begin{tabular}{|c|c|c|c|c|c|c|c|}
\hline & $\begin{array}{c}J_{l} \\
{[\mathrm{~min}]}\end{array}$ & $\begin{array}{c}J_{2} \\
{[\mathrm{~min}]}\end{array}$ & $\begin{array}{c}J_{3} \\
{[\mathrm{~min}]}\end{array}$ & $\begin{array}{c}J_{4} \\
{[\mathrm{~min}]}\end{array}$ & $\begin{array}{c}J_{5} \\
{[\mathrm{~min}]}\end{array}$ & $\begin{array}{c}J_{6} \\
{[\mathrm{~min}]}\end{array}$ & $\begin{array}{c}J_{7} \\
{[\mathrm{~min}]}\end{array}$ \\
\hline$R_{1}$ & 12 & 20 & 20 & 25 & 30 & 28 & 30 \\
\hline$R_{2}$ & 15 & 22 & 25 & 23 & 25 & 23 & 25 \\
\hline$R_{3}$ & 14 & 20 & 20 & 20 & 28 & 25 & 35 \\
\hline$R_{4}$ & 15 & 25 & 26 & 25 & 32 & 25 & 30 \\
\hline$R_{5}$ & 13 & 20 & 18 & 23 & 25 & 24 & 30 \\
\hline$R_{6}$ & 15 & 18 & 26 & 24 & 30 & 25 & 27 \\
\hline$R_{7}$ & 18 & 20 & 25 & 20 & 35 & 30 & 35 \\
\hline
\end{tabular}

In TABLE I., $R_{i}(i=1,2,3,4,5,6,7)$ represent the seven workers, $J_{i}(i=1,2,3,4,5,6,7)$ represent the seven jobs.

If each worker can finish not more than one job, the question is how to find the best assignment scheme.

\section{B. Optimize the problem with the improved Hungary algorithm}

(1) Determine virtual job and calculate the MVWT of each person.

Analyzing Fig .2, it is shows that the parallel link of the system consists of $\mathrm{J} 3, \mathrm{~J} 4$ and $\mathrm{J} 5$. Assuming that $\mathrm{JV}$ represents the virtual job:

$$
J_{v}=\left[T_{v 1}, T_{v 2}, T_{v 3}, T_{v 4}, T_{v 5}, T_{v 6}, T_{v 7}\right]^{T}
$$

Where, Tvi (i=1, 2, 3, 4, 5, 6, 7) represent the work time of each person to finish the virtual job. Through calculating, the MVWT of each person is got and the virtual job is marked as Jv0:

$$
\begin{aligned}
J_{v 0} & =\left[T_{v 1 \text { min }}, T_{v 2 \text { min }}, T_{v 3 \text { min }}, T_{v 4 \text { min }}, T_{v 5 \text { min }}, T_{v 6 \text { min }}, T_{v 7 \text { min }}\right]^{T} \\
& =[20,23,20,25,18,24,20]^{T}
\end{aligned}
$$

(2) The efficiency matrix M0 is got with the virtual job and other jobs of the series link:

$$
M 0=\left[\begin{array}{lllll}
12 & 20 & 20 & 28 & 30 \\
15 & 22 & 23 & 23 & 25 \\
14 & 20 & 20 & 25 & 25 \\
15 & 25 & 25 & 25 & 30 \\
13 & 20 & 18 & 24 & 30 \\
15 & 18 & 24 & 25 & 27 \\
18 & 20 & 20 & 30 & 35
\end{array}\right]
$$

(3) Get the optimal allocation scheme of M0 with the traditional Hungary algorithm.

$$
\left[\begin{array}{ccccc}
12 & 20 & 20 & 28 & 30 \\
15 & 22 & 23 & 23 & 25 \\
14 & 20 & 20 & 25 & 25 \\
15 & 25 & 25 & 25 & 30 \\
13 & 20 & 18 & 24 & 30 \\
15 & 18 & 24 & 25 & 27 \\
18 & 20 & 20 & 30 & 35
\end{array}\right] \Rightarrow\left[\begin{array}{ccccc}
\theta & 2 & 2 & 5 & 5 \\
3 & 4 & 5 & \theta & 0 \\
2 & 2 & 2 & 2 & \theta \\
3 & 7 & 7 & 2 & 5 \\
1 & 2 & \theta & 1 & 5 \\
3 & \theta & 6 & 2 & 2 \\
6 & 2 & 2 & 7 & 10
\end{array}\right]
$$

The total time is 98 minutes and the optimal allocation scheme is shown as TABLE II.

TABLE II. The Optimal Alloc ATION SChEME OF M0

\begin{tabular}{|c|c|c|c|c|c|c|c|}
\hline$R_{1}$ & $R_{2}$ & $R_{3}$ & $R_{4}$ & $R_{5}$ & $R_{6}$ & $R_{7}$ & Total Time \\
\hline$J_{1}$ & $J_{6}$ & $J_{7}$ & $J_{4}$ & $J_{5}$ & $J_{2}$ & $J_{3}$ & $98[\mathrm{~min}]$ \\
\hline
\end{tabular}

(4) Judge the virtual job of the optimal allocation scheme whether can be instead of a realizable parallel link which consist of the idly persons and the person who is assigned to the virtual job.

Analysis from TABLE II and Eq. 3, this scheme requires $\mathrm{R} 3, \mathrm{R} 5$ and $\mathrm{R} 7$ finish the parallel jobs within 18 minutes. However, these three workers need 25 minutes at least to finish J3, J4 and J5. Therefore, the scheme is not a realizable scheme and the time of the virtual job (Tv5) should be increased ( $18 \mathrm{~min} \rightarrow 19 \mathrm{~min})$ to get a new virtual job Jv1:

$$
J_{v 1}=[20,23,20,25,19,24,20]^{T}
$$

Make $\mathrm{Jv}_{\mathrm{V}}=\mathrm{Jv} 1$ and go to (2). This process will be repeated iteration until get the realizable optimal scheme.

\section{Results and Analysis}

After 25 iterations, the realizable optimal solution is got and shown as TABLE III. The total time of the scheme is 105 minutes and the virtual job is $\mathrm{JV}^{*}$ :

$$
J_{v^{*}}=[24,23,26,26,25,24,26]^{T}
$$

TABLE III. THE REALIZABLE OPTIMAL SCHEME

\begin{tabular}{|c|c|c|c|c|c|c|c|}
\hline$R_{1}$ & $R_{2}$ & $R_{3}$ & $R_{4}$ & $R_{5}$ & $R_{6}$ & $R_{7}$ & Total Time \\
\hline$J_{1}$ & $J_{7}$ & $J_{6}$ & $J_{4}$ & $J_{5}$ & $J_{2}$ & $J_{3}$ & $105[\mathrm{~min}]$ \\
\hline
\end{tabular}

In order to verify whether the algorithm can get the global optimal solution, this paper calculate all the possible schemes (5040 in total) with MATLAB program and the optimal scheme is shown as TABLE IV. 
TABLE IV. THE BEST OPTIMAL RESULT BY MATLAB PROGRAM

\begin{tabular}{|c|c|c|c|c|c|c|c|c|}
\hline No. & $R_{1}$ & $R 2$ & $R 3$ & $R 4$ & $R 5$ & $R 6$ & $R 7$ & $\begin{array}{c}\text { Total } \\
\text { Time }\end{array}$ \\
\hline 1 & $J_{1}$ & $J 7$ & $J_{4}$ & $J_{6}$ & $J_{5}$ & $J_{2}$ & $J_{3}$ & $105[\mathrm{~min}]$ \\
\hline 2 & $J_{1}$ & $J_{7}$ & $J_{3}$ & $J_{6}$ & $J_{5}$ & $J_{2}$ & $J_{4}$ & $105[\mathrm{~min}]$ \\
\hline $\mathbf{3}$ & $J_{1}$ & $J_{7}$ & $J_{6}$ & $J_{4}$ & $J_{5}$ & $J_{2}$ & $J_{3}$ & $\mathbf{1 0 5}[\mathbf{m i n}]$ \\
\hline
\end{tabular}

By comparing TABLE III with TABLEIV, it can be found that this method get the global optimal solution.

\section{CONCLUSION}

This paper proposed an improved Hungary algorithm to solve those problems which consist of series and parallel link. The method not only keeps the good characteristic of traditional Hungary algorithm, such as simple, but also can be brought to solve parallel link problems. The improved algorithm expands the application range of traditional Hungary algorithm.

\section{REFERENCES}

[1] Y.M. Chen. Journal of Li Shui University, Vol. 33 (2011) No.5, p 78-81.

[2] J.L. Du and J. Zhou. Value Engineering, (2010) No.3, p 120-122.
[3] T. Chang and Z.G. Han. Journal of Information Engineering University, Vol. 5 (2004) No.1, p 60-63.

[4] X.N. Ma. Journal of Chongqing Techno Business University, Vol. 31 (2014), No.12, p 68-71.

[5] W.L. Zhao. Model and Algorithm for the Irregular Flight Emergency Scheduling (MS., Civil Aviation University of China, China, 2014), p 21-31.

[6] Y. Qiu. Study on Scheduling Algorithm with Deteriorating Jobs and Multiple Maintenance Acticity (Zhejiang Gongshang University, China, 2013), p 22-47.

[7] W. Gu. A Study and Application of Target Allocation Based on Evolution Hungary Algorithm (XiDian University, China, 2013), p 37-50.

[8] S.S. Dong. The Method of High Density Cells' Tracking Based on Topological Constraint Combined with Hungarian Algorithm (Harbin Engineering University, China, 2011), p 31-43.

[9] J.X. Ren and F.J. He. Journal of Jiangxi University of Science and Technology, Vol. 35 (2014) No.3, p 63-67.

[10] J.L Li. Research on Weapon-Target Dynamic Assignment and Effectiveness Evaluation (Nanchang Hangkong University, China, 2014), p 23-27.

[11] Y.Q Song. Science \& Technology Vision, (2012) No.14, p 106108 . 